International Journal of Pure and Applied Mathematics

Volume 87 No. $6 \quad 2013,855-862$

ISSN: 1311-8080 (printed version); ISSN: 1314-3395 (on-line version)

url: http://www.ijpam.eu

doi: http://dx.doi.org/10.12732/ijpam.v87i6.14

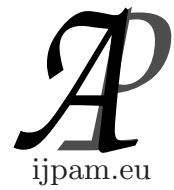

\title{
ACYCLIC EDGE-COLORING OF SIERPINSKI-LIKE GRAPHS
}

\author{
D. Paul ${ }^{1}$, Indra Rajasingh ${ }^{2}$ \\ ${ }^{1,2}$ School of Advanced Sciences \\ VIT University \\ Chennai Campus, INDIA
}

\begin{abstract}
An acyclic edge coloring of a graph is a proper edge coloring such that there are no bichromatic cycles. The acyclic chromatic index of a graph is the minimum number $k$ such that there is an acyclic edge coloring using $k$ colors and is denoted by $\chi_{a}^{\prime}(G)$. Sierpinski graphs $S(n, 3)$ are the graphs of the Tower of Hanoi with $n$ disks, while Sierpinski gasket graphs $S_{n}$ are the graphs naturally defined by the finite number of iterations that lead to the Sierpinski gasket. Sierpinski graph and Sierpinski gasket constitute Sierpinski-like graphs. We give algorithms for coloring the Sierpinski-like graphs acyclically using optimal set of colors.
\end{abstract}

AMS Subject Classification: $05 \mathrm{C} 15$

Key Words: acyclic edge-coloring, acyclic chromatic index, sierpinski graph, sierpinski gasket

\section{Introduction}

All graphs we consider are simple and finite. Let $\Delta(G)$ denote the maximum degree of a graph $G$. A coloring of the edges of a graph is proper if no pair of incident edges receive the same color. A proper coloring $C$ of the edges of a graph $G$ is acyclic if there is no two-colored (bichromatic) cycle in $G$ with respect to $C$. In other words, the subgraph induced by the union of any two color classes in $C$ is a forest. The minimum number of colors required to edgecolor a graph $G$ acyclically is termed the acyclic chromatic index of $G$ and is denoted by $\chi_{a}^{\prime}(G)$. The notion of acyclic coloring was introduced by Grunbaum

Received: September 6, 2013

(c) 2013 Academic Publications, Ltd. url: www.acadpubl.eu 
[4]. Determining $\chi_{a}^{\prime}(G)$ either theoretically or algorithmically even for special classes of graphs has been a very difficult problem [1].

The acyclic chromatic index and its vertex analogue can be used to bound other parameters like oriented chromatic number and star chromatic number of a graph G, both of which have many practical applications such as in wavelength routing in optic networks [2]. In all-optical networks a single physical optical link can carry several logical signals provided that they are transmitted on different wavelengths. An all-to-all routing in an $n$-node network is a set of $n(n-1)$ simple paths specified for every ordered pair $(x, y)$ of nodes. The routing will be feasible if an assignment of wavelengths to the paths can be given such that no link will carry in the same direction two different paths of the routing on the same wavelength.

Graphs of Sierpinski type appear naturally in many different areas of mathematics as well as in several other scientific fields. One of the most important families of such graphs is formed by the Sierpinski gasket graphs-the graphs obtained after a finite number of iterations that in the limit give the Sierpinski gasket[6]. These graphs were introduced already in 1944 by Scorer, Grundy and Smith [11]. Among others, the Sierpinski gasket graphs play an important role in dynamic systems and probability [5, 7], as well as in psychology [8].

Sierpinski gasket graphs are just a step from the Sierpinski graphs $S(n, 3)$. The graph $S_{n}$ is obtained from $S(n, 3)$ by contracting every edge of $S(n, 3)$ that lies in no triangle. One of the main features of the graphs $S(n, 3)$ is that they are precisely the graphs of the Tower of Hanoi puzzle with $n$ discs.

The graphs $S(n, 3)$ were generalized to the Sierpinski graphs $S(n, k)$ for $k \geq 3$. The motivation for this generalization came from topological studies of the Lipscombs space [9].

In this paper we determine a lower bound for $\chi_{a}^{\prime}(G)$ and prove that the bound is sharp for Sierpinski-like graphs.

\section{Sierpinski Graph}

The Sierpinski graphs $S(n, 3), n \geq 1$, are defined in the following way: $V(S(n, 3))=\{1,2,3\}^{n}$, two different vertices $u=\left(u_{1}, \ldots, u_{n}\right)$ and $v=\left(v_{1}, \ldots, v_{n}\right)$ being adjacent if and only if there exists an $h \in 1, \ldots, n$ such that:

(i) $u_{t}=v_{t}$, for $t=1, \ldots, h-1$;

(ii) $u_{h} \neq v_{h}$; and

(iii) $u_{t}=v_{h}$ and $v_{t}=u_{h}$ for $t=h+1, \ldots, n$. 


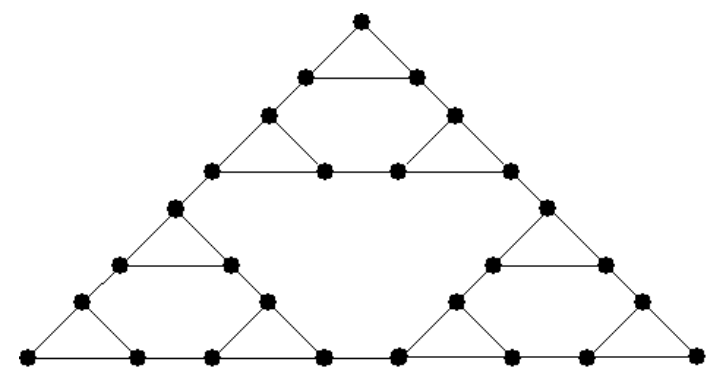

Figure 1. Sierpinski graph $S(3,3)$

Consider $\mathrm{S}(2,3)$. It contains three copies of $\mathrm{S}(1,3)$ and the bridge edges that connect each $\mathrm{S}(1,3)$ is denoted by BL,BR and BB for Left, Right and Bottom respectively. Let us name the three copies of $\mathrm{S}(1,3)$ as Top, Left and Right and name the edges as TL, TR, TB, LL, LR, LB, RL, RR, RB respectively. See Figure 2.

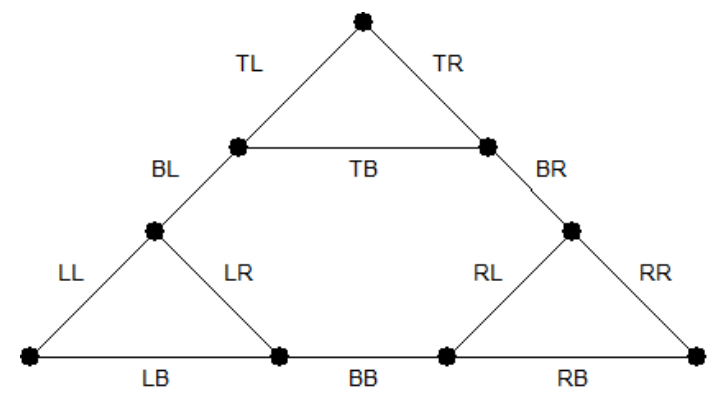

Figure 2. Labelling Sierpinski graph $S(2,3)$

\subsection{Algorithm acyclic edge-coloring (Sierpinski Graph)}

Input: Sierpinski Graph $S(n, 3)$

Algorithm:

Step 1: Arbitrarily color the edges TL, TR, TB of the top $S(1,3)$.

Step 2: $\mathrm{TL}=\mathrm{LR}=\mathrm{RB} ; \mathrm{TR}=\mathrm{LB}=\mathrm{RL} ; \mathrm{TB}=\mathrm{LL}=\mathrm{RR}$;

Step 3: $\mathrm{BL}=\mathrm{TR} ; \mathrm{BR}=\mathrm{TL} ; \mathrm{BB}=\mathrm{TB}$;

In order to know the color of the edges of the bridge and top $\mathrm{S}(1,3)$ in the next level. 

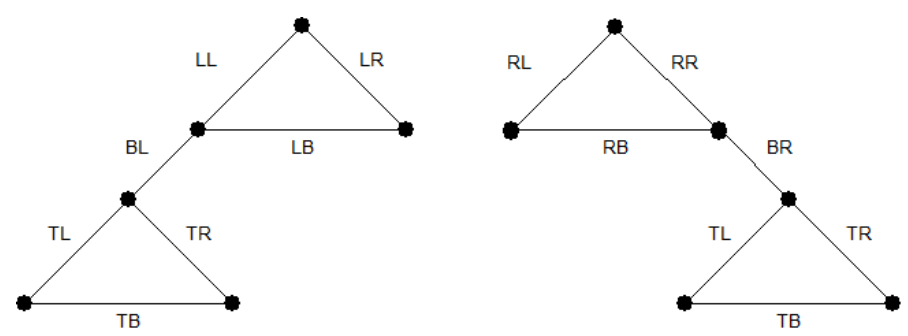

Figure 2.(a) and 2.(b)

(i) $\mathrm{BL}=\mathrm{LR} ; \mathrm{BR}=\mathrm{RL} ; \mathrm{BB}=\mathrm{LL}=\mathrm{RR}$;

(ii) Left : $\mathrm{LL}=\mathrm{TR}$; $\mathrm{LB}=\mathrm{TL} ; \mathrm{LR}=\mathrm{TB}$; See figure 2.(a).

(iii) Right: $\mathrm{RL}=\mathrm{TB} ; \mathrm{RR}=\mathrm{TL} ; \mathrm{RB}=\mathrm{TR}$; See figure 2.(b).

Repeat: Step 2

Output: $\chi_{a}^{\prime}(S(n, 3))=3$.

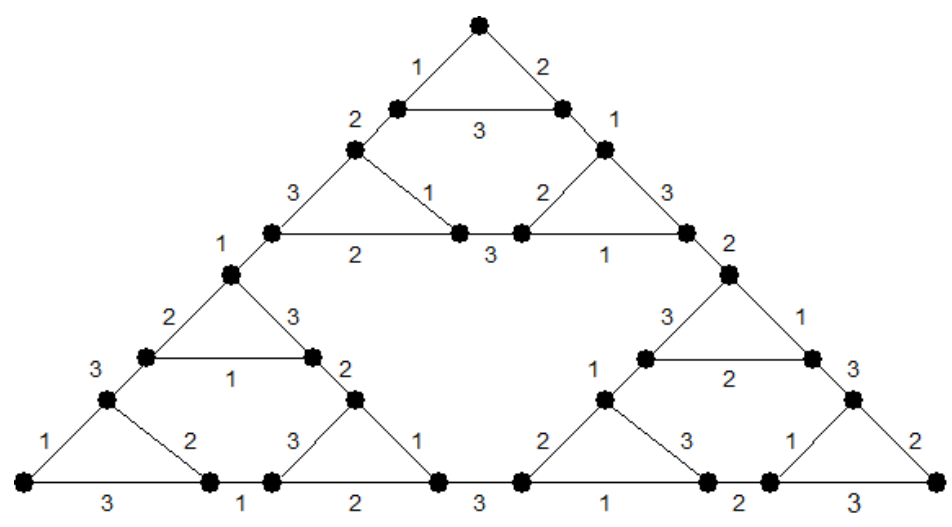

Figure 3. Acyclic Edge-coloring Sierpinski graph $S(3,3)$

\section{Proof of Correctness:}

Every cycle includes at least one oblique acute, one oblique obtuse and one horizontal edge and every cycle should pass through the bridge-edges. By the algorithm these 3 colors are different. Thus the edges of any cycle are colored with at least three colors.

We have $\chi_{a}^{\prime}(S(n, 3)) \geq \Delta(S(n, 3))=3$. By the algorithm, $\chi_{a}^{\prime}(S(n, 3))=3$. 


\section{Sierpinski Gasket}

The Sierpinski gasket graphs $S_{n}, n \geq 1$, are defined geometrically as the graphs whose vertices are the intersection points of the line segments of the finite Sierpinski gasket $\sigma_{n}$ and line segments of the gasket as edges.

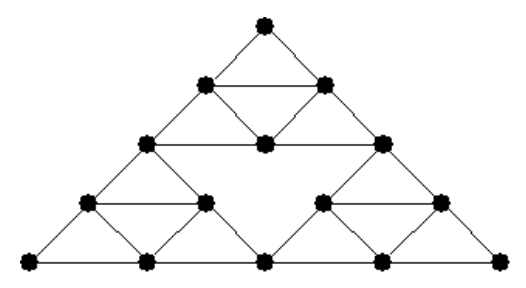

Figure 4. Sierpinski gasket $S(3)$

Consider $\mathrm{S}(2)$. It contains three copies of $\mathrm{S}(1)$, let us name the three copies of S(1) as Top, Left and Right and name the edges as TL, TR, TB, LL, LR, LB, RL, RR, RB respectively. See Figure 5 .

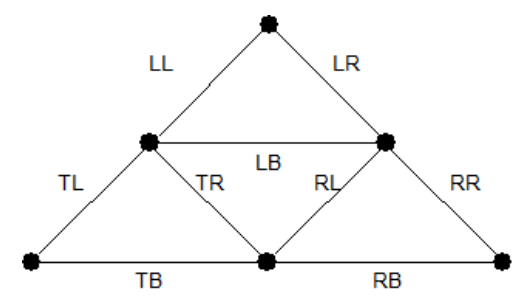

Figure 5. Labelling Sierpinski gasket $S(2)$

We should color each of the $S(2)$ using Breath-First Search(BFS) method depending on whether it is odd or even.

\subsection{Algorithm acyclic edge-coloring (Sierpinski Gasket)}

Input: Sierpinski Gasket $S(n)$

Algorithm:

Step 1: Arbitrarily color the edges TL, TR, TB of the top $S(1)$

Step 2: $n$ odd : $\mathrm{TL}=\mathrm{RR}=\mathrm{LB} ; \mathrm{TR}=\mathrm{LR} ; \mathrm{TB}=\mathrm{RB} ; \mathrm{LL}=\mathrm{RL}$ (Missing color of the four degree)

Step 3: $n$ even: $\mathrm{TR}=\mathrm{LL}=\mathrm{RB} ; \mathrm{TL}=\mathrm{RL} ; \mathrm{TB}=\mathrm{LB} ; \mathrm{LR}=\mathrm{RR}$ (Missing color of the four degree) 
In order to know the color the edges of the top $S(1)$ in the next level.
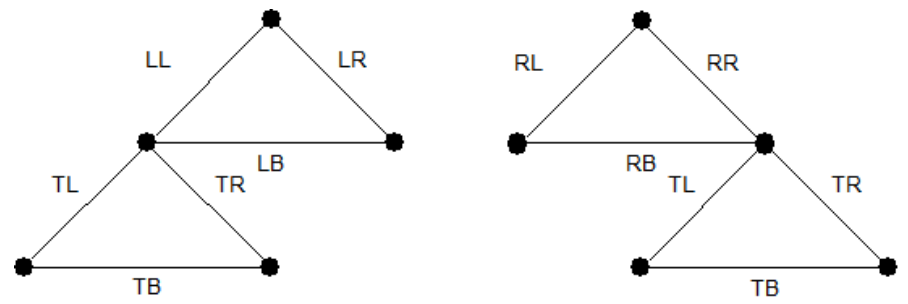

Figure 5.(a) and 5.(b)

(i) Left : $\mathrm{LR}=\mathrm{TL} ; \mathrm{LB}=\mathrm{TB}$; $\mathrm{TR}=$ Missing color of the four degree.

See figure 5.(a).

(ii) Right: $\mathrm{RL}=\mathrm{TR} ; \mathrm{RB}=\mathrm{TB}$; $\mathrm{TL}=$ Missing color of the four degree.

See figure 5.(b).

Repeat: Step 2

Output: $\chi_{a}^{\prime}(S(n))=4$.

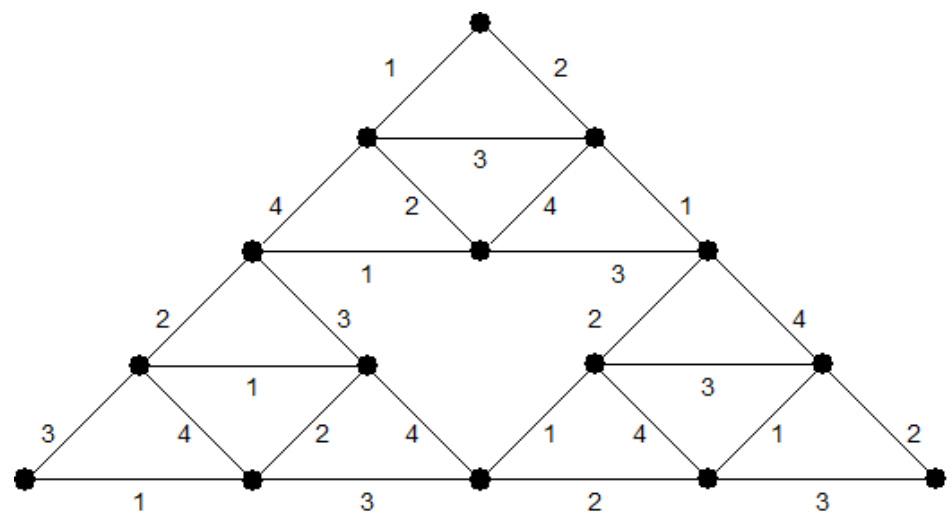

Figure 6. Acyclic Edge-coloring Sierpinski gasket $S(3)$

\section{Proof of Correctness:}

Every cycle includes at least one oblique acute, one oblique obtuse and one horizontal edge. By the algorithm these 3 colors are different. Thus the edges of any cycle are colored with at least three colors.

We have $\chi_{a}^{\prime}(S(n)) \geq \Delta(S(n))=4$. By the algorithm, $\chi_{a}^{\prime}(S(n))=4$.

The following theorem is an easy consequence of algorithm 2.1 and algorithm 3.1. 
Theorem. Let $G$ be any Sierpinski-like graphs, then $\chi_{a}^{\prime}(G)=\Delta(G)$.

\section{Conclusion}

In this paper we prove that for Sierpinski-like graphs, $\chi_{a}^{\prime}(G)=\Delta(G)$. The acyclic edge-coloring problem for Extended-Sierpinski graphs is under investigation.

\section{References}

[1] N.Alon, and A.Zaks, Algorithmic aspects of acyclic edge colorings, Algorithmica, 32 (2012), 611-614.

[2] D.Amar, A.Raspaud, and O.Togni, All to all wavelength routing in alloptical compounded networks, Discrete Mathematics, 235 (2001), 353-363.

[3] M.Basavaraju, L.S.Chandran, A note on acyclic edge coloring of complete bipartite graphs, Discrete Math., 309 (2009), 4646-4648.

[4] B.Grunbaum, Acyclic colorings of planar graphs, Israel J Math, 14 (1973), 390-408.

[5] A. M. Hinz and A. Schief, The average distance on the Sierpinski gasket, Probab. Theory Related Fields, 87 (1990), 129-138.

[6] A. Jonsson, A trace theorem for the Dirichlet form on the Sierpinski gasket, Math. Z., 250 (2005), 599-609.

[7] V. A. Kaimanovich,Random walks on Sierpi'nski graphs: hyperbolicity and stochastic homogenization, Fractals in Graz, 2001 (2003), 145-183.

[8] F. Klix and K. Rautenstrauch-Goede, Struktur- and Komponentenanalyse von Problemlosungsprozessen, Z. Psychol, 174 (1967), 167-193.

[9] S. L. Lipscomb and J. C. Perry, Lipscomb's L(A) space fractalized in Hilbert's $l^{2}(A)$ space, Proc. Amer. Math. Soc., Proc. Amer. Math. Soc., 115 (1992), 1157-1165.

[10] R.Muthu, R.Narayanan, and C.R.Subramanian, Optimal acyclic edge colouring of grid like graphs, Lecture Notes in Computer Science,Springer, 4112 (2006), 360-367. 
[11] R. S. Scorer, P. M. Grundy and C. A. B. Smith, Some binary games, Math. Gaz., 28 (1944), 96-103. 